\title{
LncRNA HAND2-AS1 overexpression inhibits cancer cell proliferation in melanoma by downregulating ROCK1
}

\author{
PENGCHENG LIU ${ }^{1,2}$, RUILI DU ${ }^{3}$ and XIN YU ${ }^{1,2}$ \\ ${ }^{1}$ Department of Hand and Pediatric Surgery, The First Hospital of Jilin University; \\ ${ }^{2}$ Jilin Province Key Laboratory of Tissue Repair, Reconstruction and Regeneration, Changchun, Jilin 130021; \\ ${ }^{3}$ Department of Clinical Laboratory, The Second Hospital of Changchun City, Changchun, Jilin 130022, P.R. China
}

Received September 11, 2018; Accepted January 1, 2019

DOI: $10.3892 / \mathrm{ol} .2019 .10402$

\begin{abstract}
Long non-coding (lnc)RNA heart and neural crest derivatives expressed 2-antisense RNA 1 (HAND2-AS1), an lncRNA antisense transcript adjacent to the heart and neural crest derivatives expressed 2 protein (HAND2), inhibits the development of several types of cancer; however, its role in melanoma is currently unknown. In the present study, it was revealed that IncRNA HAND2-AS1 was downregulated, whereas Rho-associated protein kinase 1 (ROCK1) mRNA was upregulated in tumor tissues when compared with the healthy tissues of patients with melanoma. Expression levels of IncRNA HAND2-AS1 and ROCK1 were inversely correlated in tumor tissues, but not in healthy tissues. LncRNA HAND2-AS1 expression levels were affected by tumor thickness, but not by tumor metastasis. LncRNA HAND2-AS1 overexpression led to inhibited melanoma cancer cell proliferation and an increased expression level of ROCK1. ROCK1 overexpression caused no evident effects on IncRNA HAND2-AS1 expression, but promoted cancer cell proliferation and decreased the effects of lncRNA HAND2-AS1 overexpression on cancer cell proliferation. Thus, it is possible that IncRNA HAND2-AS1 overexpression leads to inhibited cancer cell proliferation in melanoma cells through the downregulation of ROCK1.
\end{abstract}

\section{Introduction}

Melanoma is a type of malignant tumor that develops from melanocytes, which are known as pigment-containing cells (1). Melanoma is one of the most common types of cancer in the USA with an incidence rate of 1 in 100 (2). By contrast, the incidence rate of the disease in China is relatively low, with

Correspondence to: Dr Xin Yu, Department of Hand and Pediatric Surgery, The First Hospital of Jilin University, 71 Xinmin Street, Changchun, Jilin 130021, P.R. China

E-mail: bqzce06@163.com

Key words: melanoma, long non-coding RNA, heart and neural crest derivatives expressed 2-antisense RNA 1, Rho-associated protein kinase 1 only 3 in 20,000 developing this malignancy during their life-time (3). Survival conditions of patients with early-stage melanoma following surgical resection are generally satisfactory (4); however, those patients with melanoma at advanced stages of the disease typically exhibit a high prevalence of brain metastases, which is a major cause of mortality $(5,6)$. Furthermore, surgical resection of the primary tumor affects the appearance of patients, which in turn affects their quality of life (7).

As a member of the Rho-associated protein kinase (ROCK) family, ROCK1 regulates cell contraction by regulating calcium ion intake (8). Previous studies have indicated that ROCK1 also serves a critical role in the development of different types of cancer, including melanoma $(9,10)$. As a result, inhibition of ROCK1 expression is considered to be a promising therapeutic target for cancer treatment $(10,11)$. Previous studies have indicated that ROCK1 participates in cancer biology not only by interacting with proteins, but also through crosstalk with non-coding RNAs, such as long non-coding (lnc)RNAs $(12,13)$. LncRNA heart and neural crest derivatives expressed 2 -antisense RNA 1 (HAND2-AS1), an lncRNA antisense transcript adjacent to the heart and neural crest derivatives expressed 2 protein (HAND2), is a well-characterized tumor suppressor in several types of malignancy (14-16); however, its involvement in melanoma is currently unknown. Therefore, the present study aimed to investigate the involvement of HAND2-AS1 in melanoma.

\section{Materials and methods}

Cell lines and human tissues. The two human melanoma cell lines C32 and SK-MEL-28 were purchased from the American Type Culture Collection (ATCC; Manassas, VA, USA). The two cell lines were cultured in ATCC-formulated Eagle's minimum essential medium (catalog no. 30-2003) supplemented with fetal bovine serum to a concentration of $10 \%$ in an incubator at $37^{\circ} \mathrm{C}$ containing $5 \% \mathrm{CO}_{2}$.

Tumor tissue and adjacent healthy tissue (within $2 \mathrm{~cm}$ around tumors) biopsies were obtained from 56 patients with melanoma who were admitted to The First Hospital of Jilin University (Changchun, China) between May 2015 and June 2018. Tumor tissues and adjacent healthy tissues 
were examined and confirmed by three experienced pathologists. The inclusion criteria were as follows: i) Patients diagnosed via pathological biopsies; ii) patients had not been treated before admission; and iii) patients understood the experimental principle and were willing to participate. Exclusion criteria were: i) Patients diagnosed with multiple diseases, such as chronic diseases; and ii) patients refused to donate tissue samples. The 56 patients included 31 men and 25 women, and had an age range of between 26 and 67 years, with a mean age of $47.2 \pm 5.1$ years. There were 12 cases in stage I, 16 in stage II (no metastasis), 18 in stage III (lymph node metastasis) and 10 in stage IV (distant metastasis). All patients provided written informed consent. The present study was approved by the ethics committee of The First Hospital of Jilin University.

Reverse transcription-quantitative polymerase chain reaction (RT-qPCR). RNAzol ${ }^{\circledR}$ RT RNA Isolation Reagent (GeneCopoeia, Inc., Rockville, MD, USA) was used to extract total RNA from tumor tissues, adjacent healthy tissues and in vitro cultured cells. RNA concentration was determined using a NanoDrop ${ }^{\mathrm{TM}} 2000$ spectrophotometer (Thermo Fisher Scientific, Inc., Waltham, MA, USA) and reverse transcription was performed using a RevertAid RT Reverse Transcription kit (Thermo Fisher Scientific, Inc.) on the RNA samples with an $\mathrm{A}_{260} / \mathrm{A}_{280}$ ratio of between 1.8 and 2.0. The reaction conditions for reverse transcription were $25^{\circ} \mathrm{C}$ for $5 \mathrm{~min}, 50^{\circ} \mathrm{C}$ for $25 \mathrm{~min}$ and $75^{\circ} \mathrm{C}$ for $10 \mathrm{~min}$. Following these steps, a Luna ${ }^{\circledR}$ Universal One-Step RT-qPCR kit (New England BioLabs, Inc., Ipswich, MA, USA) was used for all PCRs on a CFX96 Touch ${ }^{\mathrm{TM}}$ Real-Time PCR Detection system (Bio-Rad Laboratories, Inc., Hercules, CA, USA). The reaction conditions were $95^{\circ} \mathrm{C}$ for $1 \mathrm{~min}$, then 40 cycles of $95^{\circ} \mathrm{C}$ for $10 \mathrm{sec}$ and $56.5^{\circ} \mathrm{C}$ for $42 \mathrm{sec}$. The primer sequences were 5 '-GGGTGTTTACGT AGACCAGAACC-3' (forward) and 5'-CTTCCAAAAGCC TTCTGCCTTAG-3' (reverse) for HAND2-AS1, 5'-ACC TGTAACCCAAGGAGATGTG-3' (forward) and 5'-CAC AATTGGCAGGAAAGTGG-3' (reverse) for ROCK1, and 5'-GACCTCTATGCCAACACAGT-3' (forward) and 5'-AGT ACTTGCGCTCAGGAGGA-3' (reverse) for $\beta$-actin. The $2^{-\Delta \Delta C q}$ method was used to normalize $C_{q}$ values (17).

Vectors and cell transfection. Vectors expressing HAND2-AS1 and ROCK1 were provided by GeneCopoeia, Inc. All cell transfections were performed using Lipofectamine ${ }^{\circledR} 3000$ (Invitrogen; Thermo Fisher Scientific, Inc.) with all operations performed in strict accordance with the manufacturer's protocol. Cells treated only with Lipofectamine 3000 without the presence of vectors were used as control cells. Cells transfected with empty vectors were used as negative control cells. Expression of HAND2-AS1 and ROCK1 was detected using RT-qPCR $24 \mathrm{~h}$ after transfection as aforementioned.

Cell proliferation assay. At $24 \mathrm{~h}$ following transfection, cell proliferation ability was tested using a Cell Counting Kit-8 (CCK-8) assay (Beyotime Institute of Biotechnology, Haimen, China). Briefly, cell suspensions $\left(3 \times 10^{4}\right.$ cells $\left./ \mathrm{ml}\right)$ were prepared using ATCC-formulated Eagle's minimum essential medium containing $10 \%$ fetal bovine serum. In cases of ROCK1 inhibitor treatment, cells were incubated with
$10 \mu \mathrm{M}$ ROCK1 inhibitor AT13148 (cat. no. S7563, Selleck Chemicals, Houston, TX, USA) for $24 \mathrm{~h}$ at $37^{\circ} \mathrm{C}$ before use. Each well of a 96-well plate was filled with $0.1 \mathrm{ml}$ cell suspension containing $3 \times 10^{3}$ cells. Cells were cultured in an incubator at $37^{\circ} \mathrm{C}$, containing $5 \% \mathrm{CO}_{2}$ and $10 \mu \mathrm{l} \mathrm{CCK}-8$ solution was added after 24, 28, 72 and $96 \mathrm{~h}$. Cells were cultured under the same conditions for a further $4 \mathrm{~h}$, followed by determining optical density values at $450 \mathrm{~nm}$ using a microplate reader (Bio-Rad Laboratories, Inc.).

Western blot analysis. Protein Extraction kit (catalog no. NBP2-37853; Novus Biologicals LLC, Littleton, CO, USA) was used to extract total protein from in vitro cultured cells. A bicinchoninic acid Protein Quantification kit (catalog no. ab102536; Abcam, Cambridge, UK) was used to determine protein concentrations with all steps performed in strict accordance with the manufacturer's protocol. Protein samples were denatured and subjected to SDS-PAGE (10\% gels; $45 \mu \mathrm{g}$ per lane). Following protein transfer onto polyvinylidene difluoride membranes, membranes were blocked in 5\% fat-free milk in PBS at room temperature for $2 \mathrm{~h}$. The primary antibodies used were rabbit anti-human ROCK1 (1:1,400; catalog no. ab97592; Abcam) and rabbit anti-human GAPDH, $(1: 1,400$; catalog no. ab9485; Abcam) and the incubation was performed at $4^{\circ} \mathrm{C}$ for $15 \mathrm{~h}$. A secondary incubation was performed at $24^{\circ} \mathrm{C}$ for $2 \mathrm{~h}$ using horseradish peroxidaseconjugated goat anti-rabbit immunoglobulin $\mathrm{G}$ secondary antibody (1:1,200; catalog no. MBS435036; MyBioSource, Inc., San Diego, CA, USA). An ECL ${ }^{\mathrm{TM}}$ Western Blotting Analysis system (Sigma-Aldrich; Merck KGaA, Darmstadt, Germany) was used to develop signals. ImageJ software (version 146; National Institutes of Health, Bethesda, MD, USA) was used to normalize all the data.

Statistical analysis. All experiments were performed in triplicate. Data were expressed as the mean \pm standard deviation and processed by GraphPad Prism version 6 (GraphPad Software, Inc., La Jolla, CA, USA). Correlation analyses were performed using Pearson's correlation coefficient. Comparisons between tumor tissues and adjacent healthy tissues were performed using a paired t-test. Comparisons among multiple groups were performed using one-way analysis of variance, followed by Tukey's post hoc test. $\mathrm{P}<0.05$ was considered to indicate a statistically significant difference.

\section{Results}

LncRNA HAND2-AS1 and ROCK1 mRNA expression are altered in tumor tissues compared with in adjacent healthy tissues. Expression of 1ncRNA HAND2-AS1 and ROCK1 mRNA in tumor tissues and adjacent healthy tissues of 56 patients with melanoma was detected using RT-qPCR. When compared with tumor tissues, expression levels of lncRNA HAND2-AS1 were significantly increased in adjacent healthy tissues ( $\mathrm{P}<0.05$; Fig. 1A). In contrast, expression levels of ROCK1 mRNA were significantly increased in tumor tissues compared with in healthy control tissues (P<0.05; Fig. 1B). In addition, the IncRNA HAND2-AS1 level slightly decreased (Fig. 1C), whereas the ROCK1 level slightly increased (Fig. 1D) with the increase in tumor stage. 
A

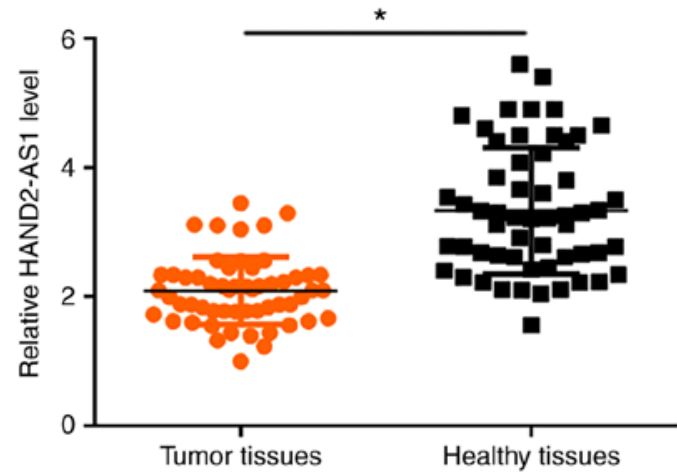

C

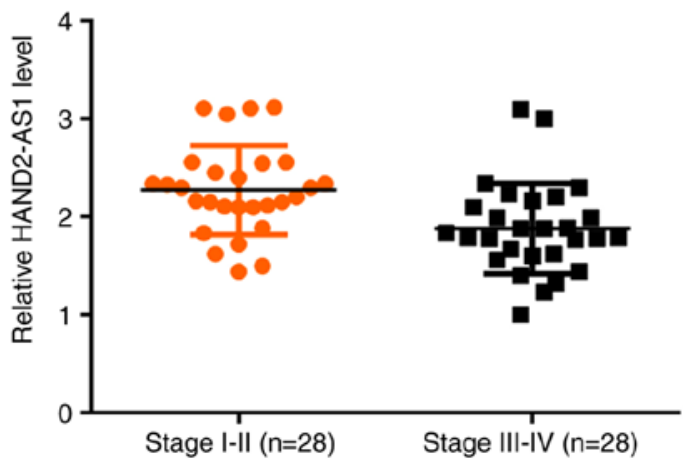

B

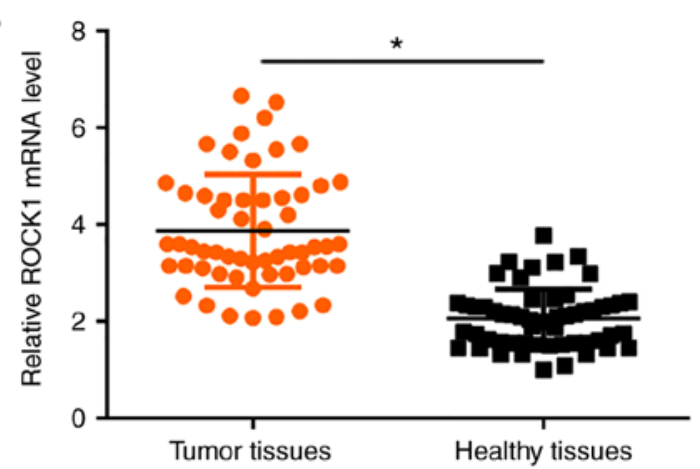

D

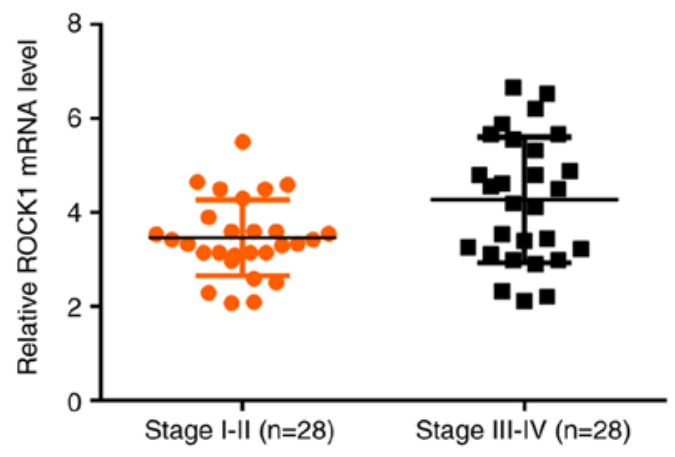

Figure 1. LncRNA HAND2-AS1 and ROCK1 mRNA expression are altered in tumor tissues compared with in adjacent healthy tissues. (A) LncRNA HAND2-AS1 was downregulated in tumor tissues compared with in adjacent healthy tissues. (B) ROCK1 mRNA was upregulated in tumor tissues compared with in adjacent healthy tissue. (C) The lncRNA HAND2-AS1 level slightly decreased with increased tumor stage. (D) The ROCK1 level slightly increased with the increase in tumor stage. "P $<0.05$. LncRNA, long non-coding RNA; HAND2-AS1, heart and neural crest derivatives expressed 2 protein-antisense RNA 1; ROCK1, Rho-associated protein kinase 1.
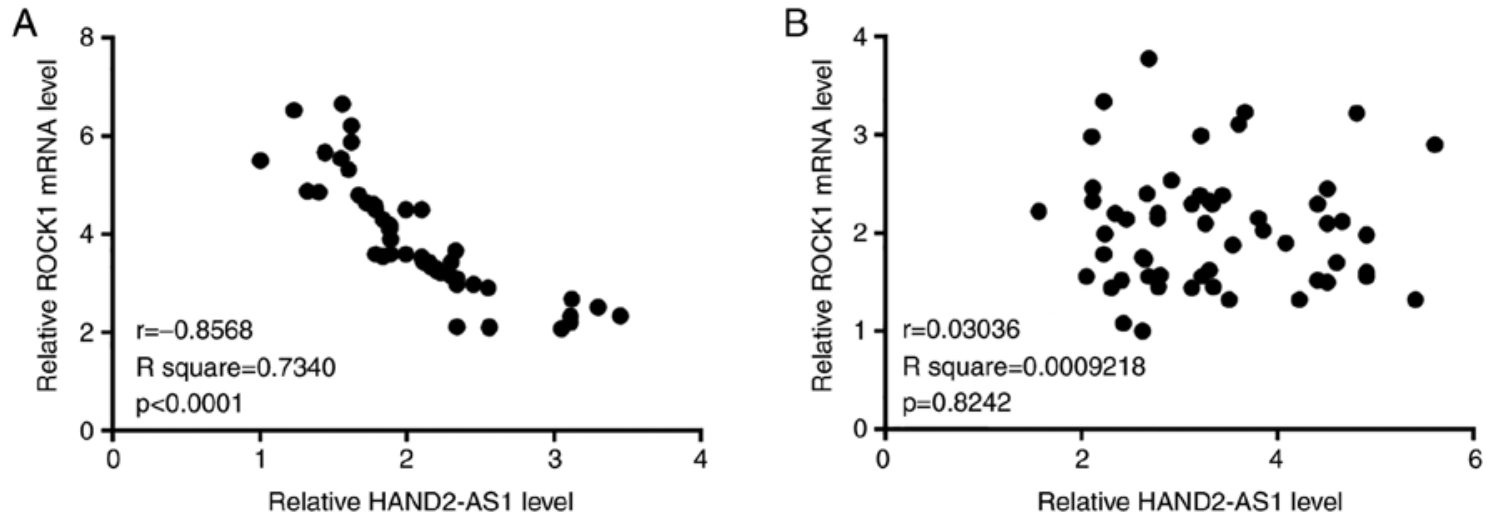

Figure 2. (A) Expression levels of lncRNA HAND2-AS1 and ROCK1 mRNA were significantly and inversely correlated in tumor tissues. (B) Expression levels of lncRNA HAND2-AS1 and ROCK1 mRNA were not significantly or inversely correlated in adjacent healthy tissues. LncRNA, long non-coding RNA; HAND2-AS1, heart and neural crest derivatives expressed 2 protein-antisense RNA 1; ROCK1, Rho-associated protein kinase 1.

Expression levels of IncRNA HAND2-AS1 and ROCK1 are inversely correlated in tumor tissues. Pearson's correlation coefficient analyses indicated that expression levels of lncRNA HAND2-AS1 and ROCK1 mRNA were significantly and inversely correlated in tumor tissues (Fig. 2A). In contrast, the correlation between expression levels of HAND2-AS1 and ROCK1 mRNA was not significant in adjacent healthy tissues (Fig. 2B).

Expression levels of IncRNA HAND2-AS1 in tumor tissues are affected by tumor thickness but not by tumor metastasis. There were 22 cases of patients with a primary tumor thickness of $<2 \mathrm{~mm}, 18$ between 2 and $4 \mathrm{~mm}$, and 16 with $>4 \mathrm{~mm}$. As presented in Fig. 3A, expression levels of IncRNA HAND2-AS1 in tumor tissues decreased with the increase in tumor thickness $(\mathrm{P}<0.05)$. There were 28 cases of non-metastasis, 18 cases of lymph node metastasis and 10 cases of distant metastasis. As presented in Fig. 3B, there were no significant differences in expression levels of lncRNA HAND2-AS1 among these three groups.

LncRNA HAND2-AS1 overexpression results in ROCK1 downregulation in cells of human melanoma cell lines. Following transfection, overexpression of HAND2-AS1 and 

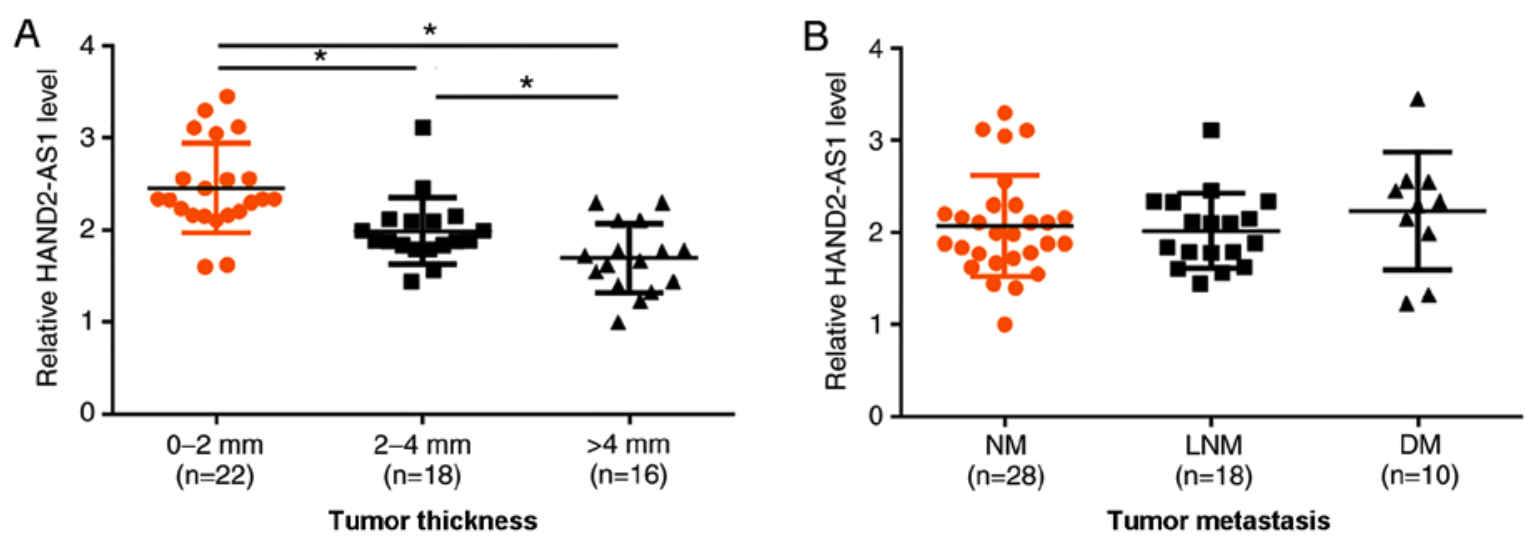

Figure 3. Expression levels of lncRNA HAND2-AS1 in tumor tissues are affected by tumor thickness but not by tumor metastasis. (A) Expression levels of lncRNA HAND2-AS1 in tumor tissues decreased as tumor thickness increased. (B) No significant differences in expression levels of lncRNA HAND2-AS1 were identified among non-metastasis, lymph node metastasis and distant metastasis groups. " $\mathrm{P}<0.05$. LncRNA, long non-coding RNA; HAND2-AS1, heart and neural crest derivatives expressed 2 protein-antisense RNA 1; NM, non-metastasis; LNM, lymph node metastasis; DM, distant metastasis.
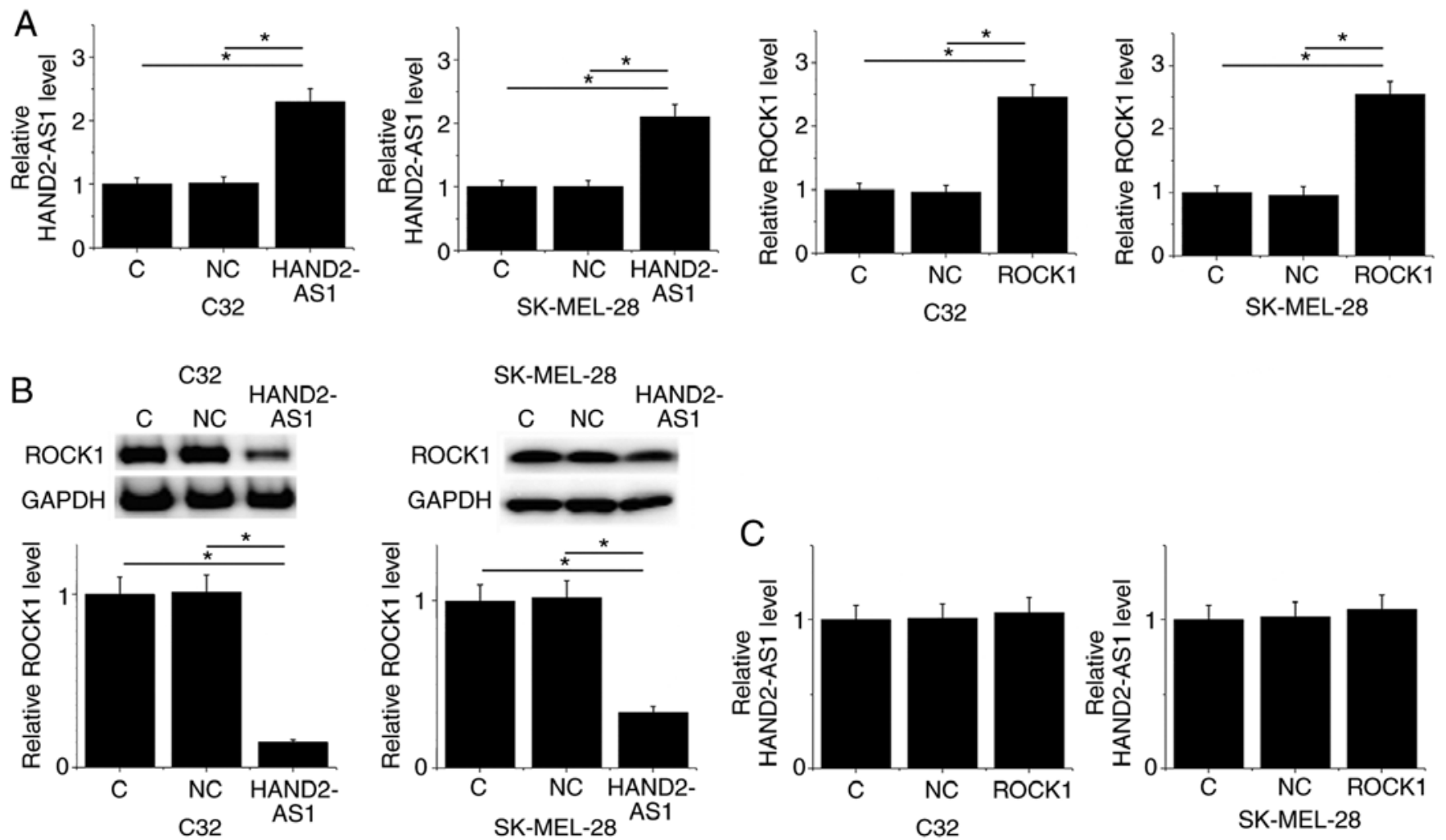

Figure 4. LncRNA HAND2-AS1 overexpression results in ROCK1 downregulation in human melanoma cell lines. (A) Overexpression of HAND2-AS1 and ROCK1 was observed in the C32 and SK-MEL-28 human melanoma cell lines. (B) HAND2-AS1 overexpression resulted in significantly downregulated ROCK1 expression in the two cell lines. (C) ROCK1 overexpression failed to significantly alter the expression level of HAND2-AS1. "P $<0.05$. LncRNA, long non-coding RNA; HAND2-AS1, heart and neural crest derivatives expressed 2 protein-antisense RNA 1; ROCK1, Rho-associated protein kinase 1; C, control; $\mathrm{NC}$, negative control.

ROCK1 was achieved in the C32 and SK-MEL-28 human melanoma cell lines $(\mathrm{P}<0.05$; Fig. 4A). Compared with the control group and negative control groups, HAND2-AS1 overexpression resulted in significantly downregulated ROCK1 expression in the two cell lines ( $\mathrm{P}<0.05$; Fig. 4B). In contrast, no significant differences in expression levels of lncRNA HAND2-AS1 were identified among the control, negative control and ROCK1 overexpression groups $(\mathrm{P}<0.05$; Fig. 4C).
LncRNA HAND2-AS1 overexpression results in inhibited proliferation of melanoma cells possibly through ROCK1. Compared with the control and negative control groups, HAND2-AS1 overexpression and treatment with ROCK1 inhibitor AT13148 resulted in clear inhibition, whereas ROCK1 overexpression led to significantly increased proliferation of C32 (Fig. 5A) and SK-MEL-28 (Fig. 5B) human melanoma cell lines $(\mathrm{P}<0.05)$. In addition, cells with HAND2-AS1 and ROCK1 overexpression exhibited significantly promoted 

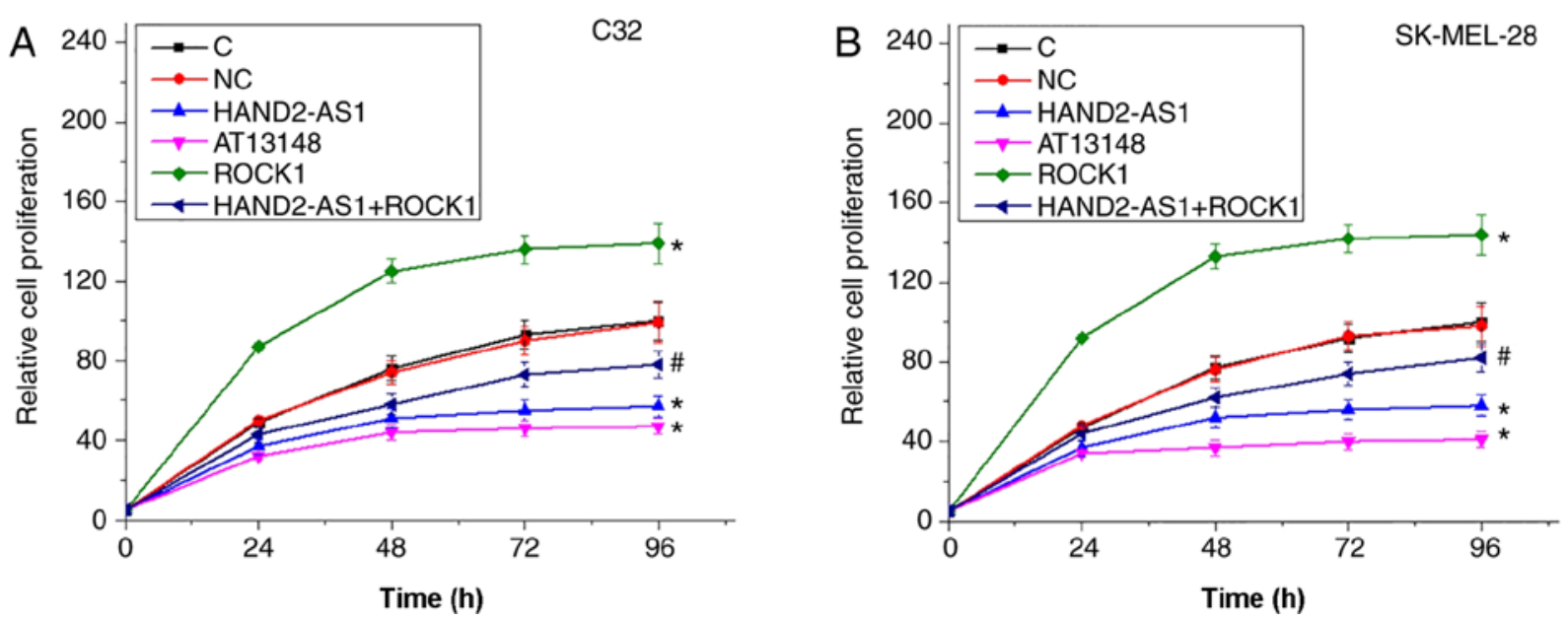

Figure 5. LncRNA HAND2-AS1 overexpression results in inhibited proliferation of melanoma cells possibly through ROCK1. (A) Cell proliferation in C32 human melanoma cell lines over time. (B) Cell proliferation in SK-MEL-28 human melanoma cell lines over time. HAND2-AS1 overexpression resulted in significant inhibition, whereas ROCK1 overexpression led to significantly increased proliferation of the two C32 and SK-MEL-28 human melanoma cell lines. In addition, ROCK1 overexpression significantly attenuated the inhibitory effects of HAND2-AS1 overexpression on cancer cell proliferation. "P $<0.05$, vs. control group; ${ }^{~} \mathrm{P}<0.05$, vs. cells with HAND2-AS1 overexpression only. LncRNA, long non-coding RNA; HAND2-AS1, heart and neural crest derivatives expressed 2 protein-antisense RNA 1; ROCK1, Rho-associated protein kinase 1; C, control; NC, negative control.

proliferation compared with cells with HAND2-AS1 overexpression alone $(\mathrm{P}<0.05)$.

\section{Discussion}

The functionality of lncRNA HAND2-AS1 has been characterized in osteosarcoma, colorectal cancer and endometrioid endometrial carcinoma (14-16). It is generally considered that lncRNA HAND2-AS1 serves a role in tumor suppression in those cancer types by inhibiting cancer progression through interactions with different signaling pathways. The key result of the present study is that IncRNA HAND2-AS1 is also a tumor suppressor in melanoma and inhibits cancer cell proliferation. The actions of IncRNA HAND2-AS1 are likely to be mediated by interactions with ROCK1.

As an oncogene, ROCK1 is typically upregulated during cancer development $(18,19)$. Consistent with previous studies $(18,19)$, the results of the present study indicated that expression of ROCK1 was significantly increased in tumor tissues compared with in adjacent healthy tissues of patients with melanoma. It has been reported that overexpression of ROCK1 is essential for the growth of certain types of tumor (20). In the present study, it was observed that ROCK1 overexpression significantly promoted the proliferation of melanoma cells, further confirming the oncogenic role of ROCK1 in melanoma.

Previous studies have indicated that differentially expressed IncRNAs are key factors in the progression of human cancer types, including melanoma $(21,22)$. As a tumor suppressor, IncRNA HAND2-AS1 has been revealed to be downregulated in osteosarcoma, colorectal cancer and endometrioid endometrial carcinoma (14-16). In the present study, it was identified that IncRNA HAND2-AS1 was significantly downregulated in tumor tissues when compared with the adjacent healthy tissues of patients with melanoma. Notably, expression of lncRNA HAND2-AS1 in tumor tissues was affected by tumor thickness, but not by tumor metastasis, indicating that lncRNA
HAND2-AS1 is unlikely to be a key regulator of cancer metastasis in melanoma. However, lncRNA HAND2-AS1 was reported previously to inhibit cancer cell invasion and metastasis in endometrioid endometrial carcinoma (16). Therefore, lncRNA HAND2-AS1 may serve a number of roles in different types of human malignancy.

In vitro cell experiments revealed that lncRNA HAND2-AS1 and ROCK1 serve opposite roles in melanoma cell proliferation. In addition, lncRNA HAND2-AS1 is likely to be an upstream inhibitor of ROCK1 in the regulation of cancer cell proliferation; however, the molecular mechanism underlying the inhibitory effects of lncRNA HAND2-AS1 on ROCK1 is currently unknown. We hypothesize that lncRNA HAND2-AS1 may inhibit ROCK1 expression through disease-associated factors due to the observation that expression levels of 1ncRNA HAND2-AS1 on ROCK1 were significantly and inversely correlated only in tumor tissues, but not in adjacent healthy tissues.

In conclusion, in the present study, lncRNA HAND2-AS1 was identified to be downregulated, and ROCK1 upregulated in melanoma. HAND2-AS1 overexpression may inhibit cancer cell proliferation in melanoma cells by downregulating ROCK1. Therefore, HAND2-AS1 overexpression may serve as a potential therapeutic target for melanoma.

\section{Acknowledgements}

Not applicable.

\section{Funding}

No funding was received.

\section{Availability of data and materials}

The datasets used and/or analyzed during the present study are available from the corresponding author on reasonable request. 


\section{Authors' contributions}

PL and XY designed the experiments; PL and RD performed the experiments and analyzed all of the data. XY drafted the manuscript and all authors approved the final manuscript.

\section{Ethics approval and consent to participate}

Ethical approval was obtained from the Ethics Committee of The First Hospital of Jilin University, Changchun, China. All procedures performed in the parts of the study involving human participants were in accordance with the ethical standards of the institutional and national research committee and with The Declaration of Helsinki (1964) and its later amendments or comparable ethical standards.

\section{Patient consent for publication}

The present study followed the tenets of The Declaration of Helsinki, and informed written consent was obtained from all patients and controls following explanation of the nature and possible consequences of the study.

\section{Competing interests}

The authors declare that they have no competing interests.

\section{References}

1. Shain AH and Bastian BC: From melanocytes to melanomas. Nat Rev Cancer 16: 345-358, 2016.

2. Miller KD, Siegel RL, Lin CC, Mariotto AB, Kramer JL, Rowland JH, Stein KD, Alteri R and Jemal A: Cancer treatment and survivorship statistics, 2016. CA Cancer J Clin 66: 271-289, 2016.

3. Chen W, Zheng R, Baade PD, Zhang S, Zeng H, Bray F, Jemal A, Yu XQ and He J: Cancer statistics in China, 2015. CA Cancer J Clin 66: 115-132, 2016.

4. Lee EH, Klassen AF, Lawson JL, Cano SJ, Scott AM and Pusic AL: Patient experiences and outcomes following facial skin cancer surgery: A qualitative study. Australas J Dermatol 57: e100-e104, 2016.

5. Khoury MN, Missios S, Tran ND and Forsyth PA: Chapter 13-Patients with brain metastases from melanoma: Treatment with surgery and radiotherapy. In: Brain Metastases From Primary Tumors 3: pp185-196, 2016.

6. Ahmed KA, Stallworth DG, Kim Y, Johnstone PA, Harrison LB, Caudell JJ, Yu HH, Etame AB, Weber JS and Gibney GT: Clinical outcomes of melanoma brain metastases treated with stereotactic radiation and anti-PD-1 therapy. Ann Oncol 27: 434-441, 2016.

7. Cromwell KD, Chiang YJ, Armer J, Heppner PP, Mungovan K, Ross MI, Gershenwald JE, Lee JE, Royal RE, Lucci A and Cormier JN: Is surviving enough? coping and impact on activities of daily living among melanoma patients with lymphoedema. Eur J Cancer Care (Engl) 24: 724-733, 2015
8. Anjum I: Calcium sensitization mechanisms in detrusor smooth muscles. J Basic Clin Physiol Pharmacol 29: 227-235, 2018.

9. Lochhead PA, Wickman G, Mezna M and Olson MF: Activating ROCK1 somatic mutations in human cancer. Oncogene 29: 2591-2598, 2010.

10. Smit MA, Maddalo G, Greig K, Raaijmakers LM, Possik PA, van Breukelen B, Cappadona S, Heck AJ, Altelaar AF and Peeper DS: ROCK1 is a potential combinatorial drug target for BRAF mutant melanoma. Mol Syst Biol 10: 772, 2014.

11. Whatcott CJ, Ng S, Barrett MT, Hostetter G, Von Hoff DD and Han H: Inhibition of ROCK1 kinase modulates both tumor cells and stromal fibroblasts in pancreatic cancer. PLoS One 12: e0183871, 2017.

12. Cui M, Wang J, Li Q, Zhang J, Jia J and Zhan X: Long non-coding RNA HOXA11-AS functions as a competing endogenous RNA to regulate ROCK1 expression by sponging miR-124-3p in osteosarcoma. Biomed Pharmacother 92: 437-444, 2017.

13. Wang $H$, Zhang $M$ and Sun G: Long non-coding RNA NEAT1 regulates the proliferation, migration and invasion of gastric cancer cells via targeting miR-335-5p/ROCK1 axis. Pharmazie 73: 150-155, 2018

14. Kang Y,Zhu X, Xu Y, Tang Q, Huang Z, Zhao Z, Lu J, Song G, Xu H, Deng $C$ and Wang J: Energy stress-induced lncRNA HAND2-AS1 represses HIF1 $\alpha$-mediated energy metabolism and inhibits osteosarcoma progression. Am J Cancer Res 8: 526-537, 2018.

15. Zhou J, Lin J, Zhang H, Zhu F and Xie R: LncRNA HAND2-AS1 sponging miR-1275 suppresses colorectal cancer progression by upregulating KLF14. Biochem Biophys Res Commun 503: 1848-1853, 2018.

16. Yang X, Wang CC, Lee WYW, Trovik J, Chung TKH and Kwong J: Long non-coding RNA HAND2-AS1 inhibits invasion and metastasis in endometrioid endometrial carcinoma through inactivating neuromedin U. Cancer Lett 413: 23-34, 2018.

17. Livak KJ and Schmittgen TD: Analysis of relative gene expression data using real-time quantitative PCR and the 2(-Delta Delta C(T)) method. Methods 25: 402-408, 2001.

18. Wang Y, Wang N, Zeng X, Sun J, Wang G, Xu H and Zhao W: MicroRNA-335 and its target Rock1 synergistically influence tumor progression and prognosis in osteosarcoma. Oncol Lett 13: 3057-3065, 2017.

19. Gilkes DM, Xiang L, Lee SJ, Chaturvedi P, Hubbi ME, Wirtz D and Semenza GL: Hypoxia-inducible factors mediate coordinated RhoA-ROCK1 expression and signaling in breast cancer cells. Proc Natl Acad Sci USA 111: E384-E393, 2014.

20. Vigil D, Kim TY, Plachco A, Garton AJ, Castaldo L, Pachter JA, Dong H, Chen X, Tokar B, Campbell SL and Der CJ: ROCK1 and ROCK 2 are required for non-small cell lung cancer anchorage-independent growth and invasion. Cancer Res 72: 5338-5347, 2012.

21. Tian Y, Zhang X, Hao Y, Fang Z and He Y: Potential roles of abnormally expressed long noncoding RNA UCA1 and Malat-1 in metastasis of melanoma. Melanoma Res 24: 335-341, 2014.

22. Wang S, Fan W, Wan B, Tu M, Jin F, Liu F, Xu H and Han P: Characterization of long noncoding RNA and messenger RNA signatures in melanoma tumorigenesis and metastasis. PLoS One 12: e0172498, 2017.

This work is licensed under a Creative Commons Attribution-NonCommercial-NoDerivatives 4.0 International (CC BY-NC-ND 4.0) License. 